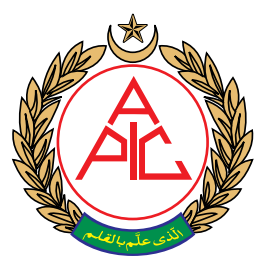

Department of Anesthesia, ICU \& Pain Management, National Cancer Institute, Cairo University, Cairo, (Egypt) Correspondence: Dr Ahmed M Soliman, Department of Anesthesia, ICU, and Pain Management, National Cancer Institute, Cairo University, Fom EL Khaleeg, Cairo, (Egypt). Tel: +201286979695 E-mail: ams21787arif@hotmail. com

Institutional Review Board (IRB), NCI approval No. IRB00004025

Received: 21 Jun 2018,

Reviewed: 25 September 2018, 2 January 2019, 6 January 2019, Revised: 4 February 2019,

Reviewed: 14 March 2019, Revised: 13 May 2019, 30 May 2019,

Accepted: 12 June 2019

\section{Ultrasound-guided assessment of diaphragmatic thickness as an indicator of successful extubation in mechanically ventilated cancer patients}

\author{
Ahmed M. Soliman, MD, Mohga A. Samy, MD, Ashraf M. Heikal, MD, \\ Mohamed A. El Ramely, MD, Tamer A. Kotb, MD
}

ABSTRACT

Objective: The study aimed to assess diaphragmatic thickness measurement by B-mode ultrasound for prediction of extubation and proper timing of weaning from mechanical ventilation in cancer patients admitted to the intensive care unit after major surgery.

Methodology: A prospective, longitudinal study conducted at Surgical ICU, National Cancer Institute, Cairo University, Cairo. Fifty patients were recruited during the immediate postoperative period after major elective cancer surgery who needed mechanical ventilation (MV). Patients were enrolled when judged to be eligible for a test of weaning from MV according to clinical and arterial blood gases (ABG) criteria. The patient was assessed for weaning according to rapid shallow breathing index (RSBI) and ultrasound guided measurements of diaphragmatic thickness ( $t d i)$ during inspiration and expiration. The percent change in tdi between end-expiration and end-inspiration $(\Delta$ tdi\%) was calculated.

The primary outcome measure was diagnostic accuracy of tdi and $\Delta$ tdi\% to predict weaning compared to ABG analysis (the gold standard for weaning).

Results: After 48 hours, 13 patients were weaned according to ABG criteria. Kappa value (agreement) between RSBI and ABG was 0.974. Kappa between both tdi and $\Delta$ tdi\% and the $A B G$ criteria was 0.891 . The values differed slightly in patients tested after 72 hours. Sensitivity of a cut off level of tdi of $2 \mathrm{~mm}$ was $84.6 \%$ and $83.3 \%$ after 48 and 72 hours of MV, respectively. Sensitivity of $\Delta$ tdi\% of $20 \%$ was clearly higher after 72 hours $(95.8 \%)$. Using ROC curves, $\Delta$ tdi\% of $>29.5 \%$ was also more sensitive after 72 hours.

Conclusion: Ultrasound estimation of diaphragm function is a promising tool to help clinicians to judge weaning readiness in patients on mechanical ventilation following major cancer surgery. Diaphragm thickness and its change between end-expiration and end-inspiration showed high degree of agreement with arterial blood gases for predicting weaning readiness.

Key words: Extubation; Diaphragm dysfunction; Diaphragm thickness; Rapid shallow breathing index; Mechanical ventilation

Abbreviations: RSBI: Rapid shallow breathing index, MV: mechanical ventilation, tdi: diaphragm thickness, $\Delta$ tdi\%: percent change in tdi between end-expiration and end-inspiration, PPV: positive predictive value, NPV: negative predictive value, kappa: measure of agreement, NCI: National Cancer Institute, VIDD: ventilator-induced diaphragmatic dysfunction

Citation: Soliman AM, Samy MA, Heikal AM, El Ramely MA, Kotb TA. Ultrasoundguided assessment of diaphragmatic thickness as an indicator of successful extubation. Anaesth pain \& intensive care 2019;23(2):178-185 


\section{INTRODUCTION}

Mechanical ventilation (MV) is a well-recognized supportive procedure for patients with respiratory failure. It is used to manage $30-40 \%$ of patients admitted to intensive care units. ${ }^{1}$ A subject of debate is what is best time for weaning from $\mathrm{MV}^{2}$ Premature $^{1,2}$ and delayed ${ }^{3,4}$ weaning, both are associated with unfavorable outcome of $\mathrm{MV}^{3,4}$ Prolonged MV increases the risk of pneumonia, and injury of the airways in addition to musculoskeletal deconditioning. Delayed weaning is associated with higher morbidity, and mortality. ${ }^{1,2}$

Identifying the patients readiness for extubation is a challenging process. ${ }^{5}$ Many studies have shown that a spontaneous breathing trial (SBT) is the most commonly used method of identifying readiness to wean. ${ }^{6,7}$ little is known about clinical outcomes and validity among the three groups in medical ICU. The objectives of this study were to evaluate the clinical relevance of weaning classification and its association with hospital mortality in a medical ICU with a protocol-based weaning program. $|n| n$ Methods $\backslash$ nAll consecutive patients admitted to the medical ICU and requiring mechanical ventilation (MV Noticeable efforts have been done to find better indices or parameters to best predict the weaning outcome. The rapid shallow breathing index (RSBI) is one of the most accurate indices that had a positive predictive value (PPV) of $85 \%{ }^{8} \mathrm{~A}$ new integrative weaning index (IWI) has been suggested that has a PPV of $99 \% .{ }^{9}$ In fact, in majority of the patients weaning is a simple affair, but weaning failure is encountered in a small number of patients and is associated with prolonged duration of MV and ICU stay and increased morbidity and mortality. ${ }^{7}$

Diaphragm dysfunction is suggested to be involved in weaning failure and is associated with negative outcome at the time of cessation of $M V^{11,12}$ Therefore, a reliable method to measure diaphragm function would be a useful guide for management of patients during $\mathrm{MV}$ and at the time of weaning. ${ }^{13}$ Ultrasonography appeared recently as a promising tool for rapid assessment of diaphragm function. ${ }^{14,15}$

In the current study, we aimed to measure the diaphragmatic thickness by B-mode ultrasound as a tool for prediction of extubation and proper timing of weaning from mechanical ventilation in cancer patients admitted to the intensive care unit after major surgery.

\section{METHODOLOGY}

This prospective study was carried out in the surgical intensive care unit at the National Cancer Institute (NCI), Cairo University. The study was approved by the institutional review board (IRB) of the NCI (approval No. IRB00004025). Fifty patients were recruited during the immediate postoperative period after major elective cancer surgery who needed mechanical ventilation (MV) due to a respiratory cause and met criteria for a spontaneous breathing trial with pressure support ventilation (PSV).

Inclusion criteria were adults of 18-65 years of age, ASA class 1 to 3, body mass index (BMI) between 20 and $40 \mathrm{~kg} / \mathrm{m}^{2}$, and duration of intubation $\geq 48$ hours. Types of abdominal cancer surgery included partial gastrectomy, hysterectomy, splenectomy, hepatectomy, and colectomy.

Exclusion criteria included hemodynamic instability requiring vasopressors, gas exchange impairment requiring positive end-expiratory pressure (PEEP) $>10 \mathrm{cmH}_{2} \mathrm{O}$ and/or $\mathrm{FIO}_{2}>50 \%$ to obtain a $\mathrm{PaO}_{2}>60 \mathrm{mmHg}$, pressure support (PS) level $>20 \mathrm{cmH}_{2} \mathrm{O}$, body temperature $>38^{\circ} \mathrm{C}$ or $<35^{\circ} \mathrm{C}$, deep sedation state (RASS score $<-1$ ), history of chronic obstructive pulmonary disease, neuromuscular disease, anatomical malformation of the diaphragm, or use of muscle paralyzing agents, aminoglycosides and corticosteroids, pneumothorax or pneumomediastinum, or increased intraabdominal pressure.

Each patient was intubated with an orotracheal tube and mechanically ventilated with PSV mode according to the clinical needs for weaning. According to the local guidelines, patients were sedated with continuous infusion of propofol started at $1.5 \mathrm{mg} / \mathrm{kg} / \mathrm{h}$ and titrated to obtain a Richmond agitation-sedation scale (RASS) score of 0/-1. Analgesia was provided by continuous epidural infusion of bupivacaine $0.125 \%$ plus fentanyl $2 \mu \mathrm{g} / \mathrm{ml}$ solution as a $6-8 \mathrm{ml} / \mathrm{h}$ to reach a numerical rating score $<4$. If epidural analgesia was not feasible, patients received 0.5 to $5 \mu \mathrm{g} / \mathrm{kg} / \mathrm{h}$ continuous IV infusion of morphine, and acetaminophen $1 \mathrm{gm} 3$ to 4 times per day.

Patients were enrolled after being judged to be eligible for a test of weaning from $\mathrm{MV}$ according to clinical and arterial blood gases (ABG) criteria. The signs included adequate cough, absence of excessive tracheobronchial secretions, clinical stability, heart rate $(\mathrm{HR})<140 / \mathrm{min}$, systolic blood pressure between 90 and $140 \mathrm{mmHg}$, respiratory rate/tidal volume ratio $<105$ breaths $/ \mathrm{min} / \mathrm{L}$. The local ICU guidelines for weaning are shown in Table 1.

Weaning trials consisted of spontaneous breathing trial (SBT) on PSV, which was adjusted until respiratory rate became 35 breaths/min or lower. The PS was reduced by $2 \mathrm{cmH}_{2} \mathrm{O}$ every 6 hours or earlier as clinically indicated. Patients were considered fit for extubation if they tolerated PS of $7 \mathrm{cmH}_{2} \mathrm{O}$ for at least 1 hour. If there were signs of intolerance, PS was increased to the preceding level and reassessment 
for weaning was performed after a period of $6 \mathrm{~h}$. A successful extubation was defined as spontaneous breathing for $>48 \mathrm{~h}$ following extubation. A failed extubation was defined as reintubation within $48 \mathrm{~h}$.

The patient was assessed for weaning clinically according to the previously mentioned guidelines, then the RSBI was calculated simultaneously with ultrasound measurements of diaphragmatic thickness during inspiration and expiration. The RSBI was calculated according to the following formula

$$
\text { tidal volume in liters (VT) }
$$

A value of 105 breaths $/ \mathrm{min} / \mathrm{L}$ or less was considered a predictor of successful extubation. ${ }^{8}$

\section{Technique of diaphragm thickness measurement:}

Diaphragm thickness $(t d i)$ was measured using a 6-13 MHz linear ultrasound probe set to B mode (SonoSite M-turbo ultrasound system with SN. WK3BKK and HFL38x/13-6 MHZ transducer with SN. 046KWD). The right hemidiaphragm was imaged at the zone of apposition of the diaphragm and rib cage in the midaxillary line between the 8th and 10th intercostal spaces. The patient was studied with the head of bed elevated 20 to 40 degrees. The tdi was measured at end-expiration and endinspiration. The first ultrasonographic measurement was performed within 24 hours after the start of MV and then daily within a $24 \pm 6 \mathrm{~h}$ time frame. The same investigator performed all the recordings. The percent change in tdi between end-expiration and end-inspiration ( $\Delta$ tdi $\%)$ was calculated as

$$
\text { ( } \left.\frac{\text { tdi end-inspiration-tdi end-expiration }}{\text { tdi end-expiration }} \times 100\right)
$$

The $\Delta$ tdi $\%$ for each patient represented the mean of three breaths. Images were obtained after 24 hours of ventilation and once per day until extubation and immediately before extubation.

\section{Diaphragm at Zone of Apposition (ZAP):}

The ZAP of the diaphragm refers to the area where the diaphragm marches with the lower rib cage (Figure 1). In this region the diaphragm is relatively very superficial structure. Once obtained, the image can be displayed in B-mode (Figure 2).

To visualize the diaphragm in the ZAP, the transducer is placed on the skin surface in the coronal plane. The diaphragm in the ZAP is best visualized in the midaxillary line in the region of the lower rib cage, usually in the 8th or 9th intercostal space. The diaphragm is identified as a three-layered structure just superficial to the liver, consists of two parallel echogenic lines (peritoneal and diaphragmatic pleura) sandwiching a relatively non-echogenic layer, the diaphragm muscle itself. On occasion, a 4th noncontinuous line may be seen within the diaphragm muscular layer which may represent a neural or vascular structure. The diaphragm can be further identified dynamically as the most superficial structure which is obliterated by the leading edge of the lung during inspiration.

Once the diaphragm is identified, the image was frozen at end-expiration and the thickness of the diaphragm (tdi) was measured as the distance from the middle of the diaphragmatic pleura to the middle of the peritoneum. A subsequent image is obtained at end-inspiration, and $t d i$ was measured. Normal values for diaphragm thickness at end-expiration generally fall between 2.0 and $3.5 \mathrm{~mm}$. Normal values for diaphragm thickening with inspiration $(\Delta \operatorname{tdi} \%)$ fall between $20 \%$ and $100 \% .^{16}$

The primary outcome measure was diagnostic accuracy of diaphragm thickness and $\Delta$ tdi\% to predict weaning compared to ABG (the gold standard for weaning). Secondary outcome measures were diagnostic accuracy of RSBI compared to ABG and duration of ventilation.

\section{Statistical analysis:}

Statistical analysis was done using IBM $\odot$ SPSS $\odot$ Statistics version 22 (IBM $\odot$ Corp., Armonk, NY, USA). McNemar test was used to examine the relation between qualitative variables.

\begin{tabular}{|c|c|}
\hline \multicolumn{2}{|c|}{ CNS: } \\
\hline$\bullet$ & Arousable \\
\hline$\bullet$ & $\mathrm{GCS}>12$ \\
\hline - & Cardiovascular: \\
\hline - & $\mathrm{HR}<140$ \\
\hline - & Not on pressors \\
\hline \multicolumn{2}{|c|}{ Others: } \\
\hline - & Afebrile \\
\hline$\bullet$ & No significant electrolyte abnormalities. \\
\hline \multicolumn{2}{|c|}{ Respiratory: } \\
\hline \multicolumn{2}{|c|}{$<8 \mathrm{Cm} \mathrm{H}_{2} \mathrm{O}$} \\
\hline - & $\mathrm{PaCO}_{2}$ at baseline \\
\hline - & Rate/Tidal Volume $<105 / \mathrm{min} / \mathrm{L}$ \\
\hline$\cdot$ & Max Inspiratory Pressure $-25 \mathrm{cmH}_{2} \mathrm{O}$ \\
\hline - & $\mathrm{PaO}_{2} / \mathrm{FiO}_{2}>200$ \\
\hline$\bullet$ & Tidal Volume $5 \mathrm{~mL} / \mathrm{kg}$ \\
\hline - & Respiratory Rate $<35 /$ min \\
\hline - & Vital Capacity $10 \mathrm{~mL} / \mathrm{kg}$ \\
\hline$\bullet$ & Minute Ventilation $<10 \mathrm{~L} / \mathrm{min}$ \\
\hline
\end{tabular}

Agreement between different diagnostic methods

Table 1: NCI protocols for weaning in ICU 


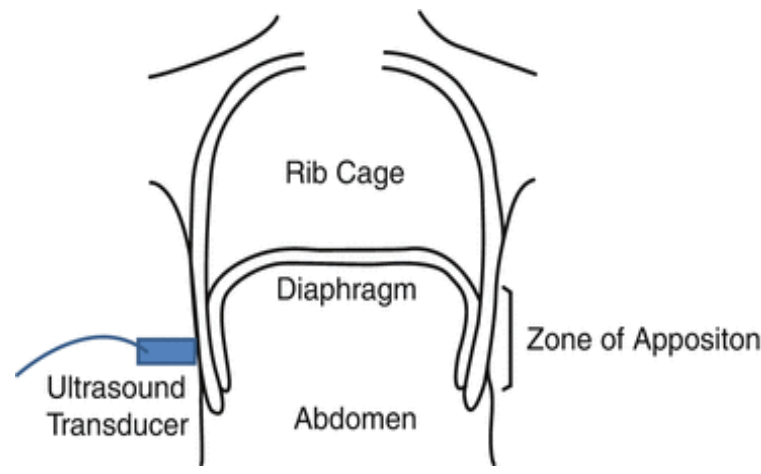

Figure 1: Schematic illustration of ultrasound transducer placement for visualizing the diaphragm in the zone of apposition ${ }^{16}$

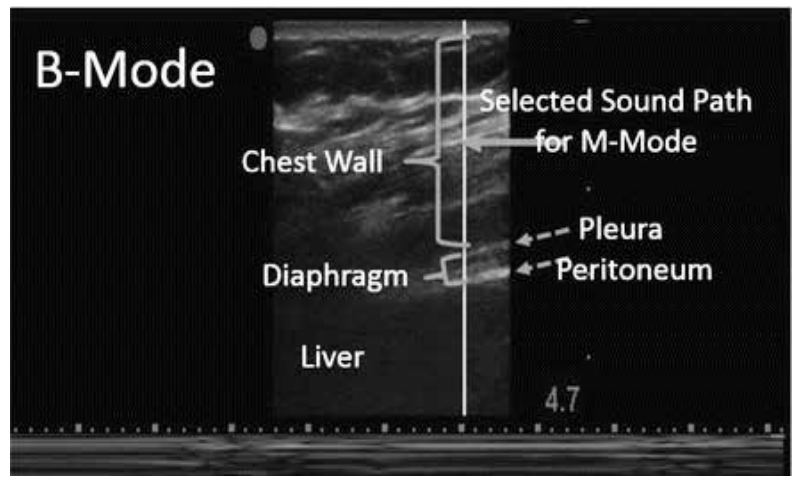

Figure 2: B-Mode of the diaphragm in the zone of apposition, the diaphragm muscle is visualized as the minimally echogenic structure "sandwiched" between the pleura and peritoneum ${ }^{16}$

was judged with kappa test. Receiver operator characteristic curve analysis was used to select the best cut-off point for different accuracy measures which included sensitivity, specificity, predictive values of both negative and positive tests. A p $<0.05$ was considered significant.

\section{RESULTS}

The mean age of the studied group was $50.6 \pm 7.6$ y. There were 23 males and 27 females. Their BMI was $31.1 \pm 4.7 \mathrm{~kg} / \mathrm{m}^{2}$. Table 2 shows the associated comorbidities and types of operations performed. Duration of ventilation and indications are shown in Table 3. The median duration of MV was 3 days (range: 2-5 days).

Two patients needed reintubation after 24 hours of weaning and after fulfilling all criteria of weaning secondary to a new incidence on top of the basic medical condition, e.g. sudden cardiac arrest due to massive pulmonary embolism in one patient
Table 2: Comorbidities and types of operations in the studied group

\begin{tabular}{l|c}
\multicolumn{1}{c|}{ Comorbidities } & No. (\%) \\
\hline None & $32(64)$ \\
\hline Hypertension & $7(14)$ \\
\hline Diabetes mellitus & $6(12)$ \\
\hline Hypertension and diabetes mellitus & $4(8)$ \\
\hline Ischemic heart disease & $1(2)$ \\
\hline Type of Operation & \\
\hline Pancreatectomy & $9(18)$ \\
\hline Radical cystectomy & $8(16)$ \\
\hline Lt Hemicolectomy & $8(16)$ \\
\hline Panhysterectomy & $8(16)$ \\
\hline Rt hemicolectomy & $7(14)$ \\
\hline Nephrectomy & $4(8)$ \\
\hline Adrenalectomy & $2(4)$ \\
\hline Distal gastrectomy & $2(4)$ \\
\hline Total colectomy & $1(2)$ \\
\hline Pan-hysterectomy, oophorectomy, & $1(2)$ \\
\hline omentectomy and lymphadenectomy & \\
\hline
\end{tabular}

and complicated surgery in the other. After $48 \mathrm{~h}$, 13 patients out of the remaining 48 were weaned according to the arterial blood gases (ABG) criteria. According to the reference value of RSBI, 12 patients were weanable and 36 were not. Therefore the kappa value (agreement) between RSBI and ABG criteria was 0.974 . On the other hand agreement between both tdi and $\Delta$ tdi $\%$ and the ABG criteria was 0.891 (Table 4). After $72 \mathrm{~h} 24 / 35$ patients were weaned according to the arterial blood gases (ABG) criteria. The values of agreement of RSBI, tdi, and $\Delta$ tdi $\%$ with ABG criteria differed slightly in patients tested after $72 \mathrm{~h}$.

Using ROC curve analysis, the suggested best cut-off of RSBI after 48 and $72 \mathrm{~h}$ was the same as previously

Table 3: Indications and duration of ventilation in the studied group

\begin{tabular}{l|c}
\multicolumn{1}{c|}{ Cause of ventilation } & No. (\%) \\
\hline Sepsis & $33(66)$ \\
\hline pneumonia & $8(16)$ \\
\hline pulmonary embolism & $5(10)$ \\
\hline Hemorrhagic Shock & $4(8)$ \\
\hline Duration of ventilation (days) ${ }^{*}$ & \\
\hline 2 & $13(27.1)$ \\
\hline 3 & $24(50.0)$ \\
\hline 4 & $9(18.8)$ \\
\hline 5 & $4(8.3)$ \\
\hline${ }^{*} n=48$. &
\end{tabular}


Table 4: Diagnostic accuracy of RSBI, tdi, and $\Delta$ tdi\% according to their reference values for prediction of successful weaning after 48 and 72 hours

\begin{tabular}{|c|c|c|c|c|c|c|}
\hline & kappa & Sensitivity & Specificity & PPV & NPV & Accuracy \\
\hline \multicolumn{7}{|c|}{ After 48 hours $(n=13 / 48)$} \\
\hline RSBI & 0.974 & $92.3 \%$ & $100.0 \%$ & $100.0 \%$ & $97.4 \%$ & $98.0 \%$ \\
\hline tdi & 0.891 & $84.6 \%$ & $100.0 \%$ & $100.0 \%$ & $94.9 \%$ & $96.0 \%$ \\
\hline$\Delta \mathrm{tdi} \%$ & 0.891 & $84.6 \%$ & $100.0 \%$ & $100.0 \%$ & $94.9 \%$ & $96.0 \%$ \\
\hline \multicolumn{7}{|c|}{ After 72 hours $(n=24 / 35)$} \\
\hline RSBI & 0.885 & $91.7 \%$ & $100.0 \%$ & $100.0 \%$ & $86.7 \%$ & $94.6 \%$ \\
\hline tdi & 0.778 & $83.3 \%$ & $100.0 \%$ & $100.0 \%$ & $76.5 \%$ & $89.2 \%$ \\
\hline$\Delta$ tdi $\%$ & 0.942 & $95.8 \%$ & $100.0 \%$ & $100.0 \%$ & $92.9 \%$ & $97.3 \%$ \\
\hline
\end{tabular}

RSBI: Rapid shallow breathing index, tdi: diaphragm thickness, $\Delta t d i \%$ : percent change in tdi between end-expiration and end-inspiration, PPV: positive predictive value, NPV: negative predictive value, kappa: measure of agreement

Table 5: Results of ROC curve analysis of tdi and $\Delta$ tdi\% in detecting weanability off mechanical ventilator after 48 and 72 hours

\begin{tabular}{l|c|c|c|c} 
& \multicolumn{2}{|c|}{$\begin{array}{c}\text { After 48 hours } \\
(\mathbf{n = 1 3 / 4 8 )}\end{array}$} & \multicolumn{2}{c}{$\begin{array}{c}\text { After 72 hours } \\
(\mathbf{n = 2 4 / 3 5 )}\end{array}$} \\
\cline { 2 - 5 } & $\begin{array}{c}\text { tdi } \\
\mathbf{1 . 9} \mathbf{~ m m}\end{array}$ & $\begin{array}{c}\mathbf{\Delta t d i} \% \\
\mathbf{2 4 . 2 \%}\end{array}$ & $\begin{array}{c}\text { tdi } \\
\mathbf{2 . 1 5} \mathbf{~ m m}\end{array}$ & $\begin{array}{c}\mathbf{\Delta} \text { tdi\% } \\
\mathbf{2 2 9 . 5 \%}\end{array}$ \\
\hline AUC & 0.946 & 0.957 & 0.934 & 0.994 \\
\hline $95 \% \mathrm{Cl}$ & $0.843-0.990$ & $0.859-0.994$ & $0.802-0.989$ & $0.893-1.000$ \\
\hline Sensitivity & $84.6 \%$ & $84.6 \%$ & $83.3 \%$ & $95.8 \%$ \\
\hline Specificity & $100.0 \%$ & $100.0 \%$ & $100.0 \%$ & $100.0 \%$ \\
\hline PPV & $100.0 \%$ & $100.0 \%$ & $100.0 \%$ & $100.0 \%$ \\
\hline NPV & $94.9 \%$ & $94.9 \%$ & $76.5 \%$ & $92.9 \%$ \\
\hline
\end{tabular}

AUC: Area under the curve, $95 \% \mathrm{Cl}$ : confidence interval, PPV: positive predictive value, NPV: negative predictive value

determined ( $\leq 105$ breaths $/ \mathrm{min} / \mathrm{L}$ ). Table 5 shows slightly different cut-off values of tdi and $\Delta$ tdi\% after 48 and $72 \mathrm{~h}$ with their diagnostic accuracy values.

\section{DISCUSSION}

Both premature and delayed liberation from MV can be hazardous to patients in the ICU. Weaning should be planned as patients who accidentally self-extubate have a $31-78 \%$ risk of reintubation. ${ }^{17}$ of whom 450 were on MV. Of the patients on MV, 30 (6.7\% Even the most experienced clinicians may misinterpret the readiness for ventilator weaning. This is why solid protocols involving multiple parameters have to be evaluated to reach a sound unhazardous clinical judgment; to wean or not to wean.

In our institution, we implement guidelines including clinical parameters in addition to clear cut-off values of arterial blood gases to decide readiness for weaning off $M V$. In the current study, based on the negative impact of diaphragm dysfunction on weaning, we tested the capability of ultrasound estimation of diaphragm function to predict successful weaning against the ABG protocol as a gold standard.

The study demonstrated that diaphragm thickness (tdi) and percent change in tdi between end-expiration and endinspiration $(\Delta$ tdi\%) were highly specific indicators of weanability of the studied patients; both had $100 \%$ positive predictive value. Sensitivity of a cut off level of tdi 2 $\mathrm{mm}$ was $84.6 \%$ and $83.3 \%$ after 48 and 72 hours of $M V$, respectively. Sensitivity of $\Delta$ tdi\% of $20 \%$ was clearly higher after 72 hours (95.8\%). Using ROC curves, $\Delta$ tdi $\%$ of $>29.5 \%$ was also more sensitive after 72 hours. Previous studies reported different cut-off of $\Delta \mathrm{tdi} \%$ to predict successful weaning that ranged from 20 to $36 \%$ depending on the ventilator support provided during measurement. ${ }^{14,15,18-21}$ rather than diaphragm motion, can be used to predict extubation success or failure.|nMethods Sixty-three mechanically ventilated patients were prospectively recruited. Diaphragm thickness (tdi

Dinino, et al. concluded that ultrasound measurement of diaphragmatic thickness can be helpful in reducing the number of failed extubation. The sensitivity and specificity of $\Delta$ tdi $\%$ at a cutoff $\geq 30 \%$ for were $88 \%$ and $71 \%$, respectively. ${ }^{14}$ In another study, the sensitivity and specificity were $82 \%$ and $88 \%$, respectively with a cut-off of $\Delta$ tdi\% $>36 .{ }^{15}$ More recently, Ali and colleagues reported a sensitivity of $\Delta$ tdi $\%>30 \%$ of $97.3 \%$ and specificity of $85.2 \%$ for successful weaning. They found that a diaphragm thickness $>2 \mathrm{~mm}$ had sensitivity of $79.3 \%$ and specificity of $77.7 \% .^{22}$

A recent meta-analysis of 13 studies involving 742 patients reported a pooled sensitivity and specificity of $\Delta$ tdi $\%$ of $89.3 \%$ and $79.6 \%$, respectively. The authors concluded that ultrasonography of the diaphragm is a promising tool to predict reintubation within 48 hours of extubation. ${ }^{23}$ Embase, and Cochrane Library to identify all the relevant papers, published in English up to July 16, 2017.

Eligible studies were included if data were in adequate details to rebuild $2 \times 2$ contingency tables. 
Methodological quality of the included studies was evaluated using the Quality Assessment of Diagnostic Accuracy Studies-2 (QUADAS-2 However, the included studies were characterized by evident heterogeneity.

Mechanical ventilation has been shown to induce diaphragm dysfunction due to loss of diaphragmatic force-generating capacity. This was termed ventilatorinduced diaphragmatic dysfunction (VIDD) ${ }^{24}$ Also, endurance of the diaphragm is also negatively affected by $\mathrm{MV}^{25}$ Hermans and colleagues ${ }^{26}$ demonstrated a logarithmic decline in diaphragmatic force correlated with the duration of MV; a finding matched with the concept of VIDD.

Ultrasound offers a readily available tool for assessment of diaphragm function during weaning. Using the intercostal approach, ultrasound enables measurement of the diaphragm thickness on its zone of apposition to the rib cage.

The change in tdi between end-expiration and endinspiration $(\Delta$ tdi\%) is strongly correlated with transdiaphragmatic pressure ${ }^{27} 10$ and $15 \mathrm{cmH}(2$, which is an estimator of diaphragm function. ${ }^{28}$

Another tool for assessment of weaning readiness in the current study was RSBI. A RSBI $\leq 105$ breaths/ $\mathrm{min} / \mathrm{L}$ had a sensitivity of $92.3 \%$ and specificity of $97.4 \%$ to predict readiness to weaning against the gold standard; the ABG. With longer duration of $M V$, the specificity decreased to $86.7 \%$. Fadaii et al. reported sensitivity of $77.8 \%$ and specificity of $71.4 \%$ of RSBI. ${ }^{29}$

More recently, Pirompanich and Romsaiyut suggested combination of RSBI with ultrasound measurement of diaphragm thickness for prediction of weaning success. They reported sensitivity of $96 \%$, specificity of $68 \%$, positive predictive value of $89 \%$, negative predictive value of $86 \%$ for prediction of successful weaning for $\Delta$ tdi $\% \geq 26 \%$. Specificity increased to $78 \%$ when $\Delta$ tdi $\% \geq 26 \%$ was combined with RSBI $\leq 105 .{ }^{30}$

\section{LIMITATIONS}

The study has few limitations. In this study, we did not asses other muscles, such as intercostal muscles, pectoralis muscles, or leg muscles as that atrophy of diaphragm may be a part of disuse atrophy. The sample size was relatively small. The ultrasound technique recommended by the study needs training for the ICU practitioners to be familiar with.

\section{CONCLUSION}

Ultrasound estimation of diaphragm function is a promising tool to help clinicians to judge weaning readiness in patients on mechanical ventilation following major cancer surgery. Diaphragm thickness and its change between end-expiration and endinspiration showed high degree of agreement with arterial blood gases for predicting weaning readiness. Both parameters had absolute specificity and positive predictive value. It is recommend to add ultrasonographic diaphragm thickness measurement to criteria of deciding liberation from MV especially with longer duration of on ventilators.

Financial support: None to declare

Conflicts of Interest: None to declare

Authors' contribution:

AMS: Conduction of the study work, manuscript editing

MAS: Concept, Final revision

AMH: Manuscript editing

MAR + TAK: Conduction of the study work 


\section{REFERENCES}

1. Esteban A, Anzueto A, Frutos $F$, Alía I, Brochard L, Stewart TE, et al. Characteristics and outcomes in adult patients receiving mechanical ventilation: a 28-day international study. JAMA. 2002;287(3):345-355. [PubMed]

2. Wawrzeniak IC, Regina Rios Vieira S, Almeida Victorino J. Weaning from Mechanical Ventilation in ARDS: Aspects to Think about for Better Understanding, Evaluation, and Management. Biomed Res Int. 2018;2018. [PubMed] [Free full text] DOI:10.1155/2018/5423639

3. Seymour CW, Martinez A, Christie JD, Fuchs BD. The outcome of extubation failure in a community hospital intensive care unit: a cohort study. Crit Care. 2004;8(5):R322-327. [PubMed] [Free full text]

4. Coplin WM, Pierson DJ, Cooley KD, Newell DW, Rubenfeld GD. Implications of extubation delay in brain-injured patients meeting standard weaning criteria. Am J Respir Crit Care Med. 2000;161(5):1530-1536. [PubMed]

5. Boles J-M, Bion J, Connors A, Herridge M, Marsh B, Melot C, et al. Weaning from mechanical ventilation. Eur Respir J. 2007;29(5):1033-1056. [PubMed]

6. Jeong BH, Ko MG, Nam J, Yoo H, Chung CR, Suh GY, et al. Differences in clinical outcomes according to weaning classifications in medical intensive care units. PLoS One. 2015;10(4). [PubMed] [Free full text] DOI:10.1371/journal.pone.0122810

7. Béduneau G, Pham T, Schortgen F, Piquilloud L, Zogheib E, Jonas $M$, et al. Epidemiology of Weaning Outcome according to a New Definition. The WIND Study. Am J Respir Crit Care Med. 2017;195(6):772783. [PubMed] DOI:10.1164/ rccm.201602-03200C

8. Yang KL, Tobin MJ. A prospective study of indexes predicting the outcome of trials of weaning from mechanical ventilation. N Engl J Med.
1991;324(21):1445-1450. [PubMed]

9. Nemer SN, Barbas CS, Caldeira JB, Cárias TC, Santos RG, Almeida LC, et al. A new integrative weaning index of discontinuation from mechanical ventilation. Crit Care. 2009;13(5):R152. [PubMed] [Free full text] DOI: 10.1186/cc8051

10. Delisle $S$, Francoeur $M$, Albert $M$, Ouellet $\mathrm{P}$, Bellemare $\mathrm{P}$, Arsenault P. Preliminary evaluation of a new index to predict the outcome of a spontaneous breathing trial. Respir Care. 2011;56(10):15001505. [PubMed] DOI: 10.4187/ respcare.00768

11. Kim WY, Suh HJ, Hong S-B, Koh Y, Lim C-M. Diaphragm dysfunction assessed by ultrasonography: influence on weaning from mechanical ventilation. Crit Care Med. 2011;39(12):26272630. [PubMed] DOI: 10.1097/ CCM.0b013e3182266408

12. Jung $B$, Moury $P H$, Mahul $M$, de Jong A, Galia F, Prades $A$, et al. Diaphragmatic dysfunction in patients with ICU-acquired weakness and its impact on extubation failure. Intensive Care Med. 2016;42(5):853-861. [PubMed] D0l:10.1007/s00134-0154125-2

13. Dubé B-P, Dres M, Mayaux J, Demiri S, Similowski T, Demoule A. Ultrasound evaluation of diaphragm function in mechanically ventilated patients: comparison to phrenic stimulation and prognostic implications. Thorax. 2017;72(9):811-818. [PubMed] D0I:10.1136/thoraxjnl-2016-209459

14. DiNino E, Gartman EJ, Sethi JM, McCool FD. Diaphragm ultrasound as a predictor of successful extubation from mechanical ventilation. Thorax. 2014;69(5):431-435. [PubMed] D0l: 10.1136/thoraxjnl-2013-204111

15. Ferrari G, De Filippi G, Elia F, Panero F, Volpicelli G, Aprà F. Diaphragm ultrasound as a new index of discontinuation from mechanical ventilation. Crit Ultrasound J. 2014;6(1):8. [PubMed] [Free full text]
DOI: $10.1186 / 2036-7902-6-8$

16. Cohn D, Benditt JO, Eveloff S, McCool FD. Diaphragm thickening during inspiration. J Appl Physiol. 1997;83(1):291-296. [PubMed]

17. Lee TW, Hong JW, Yoo J-W, Ju S, Lee SH, Lee SJ, et al. Unplanned Extubation in Patients with Mechanical Ventilation: Experience in the Medical Intensive Care Unit of a Single Tertiary Hospital. Tuberc Respir Dis (Seoul). 2015;78(4):336-340. [PubMed] [Free full text] DOI: 10.4046/ trd.2015.78.4.336

18. Farghaly S, Hasan AA. Diaphragm ultrasound as a new method to predict extubation outcome in mechanically ventilated patients. Aust Crit Care. 2017;30(1):37-43. [PubMed] DOl: 10.1016/j.aucc.2016.03.004

19. Blumhof $\mathrm{S}$, Wheeler $\mathrm{D}$, Thomas $\mathrm{K}$, McCool FD, Mora J. Change in Diaphragmatic Thickness During the Respiratory Cycle Predicts Extubation Success at Various Levels of Pressure Support Ventilation. Lung. 2016;194(4):519-525. [PubMed] DOI: 10.1007/s00408-016-9911-2

20. Dres M, Dubé B-P, Mayaux J, Delemazure J, Reuter D, Brochard L, et al. Coexistence and Impact of Limb Muscle and Diaphragm Weakness at Time of Liberation from Mechanical Ventilation in Medical Intensive Care Unit Patients. Am J Respir Crit Care Med. 2017;195(1):57-66. [PubMed] DOI:10.1164/rccm.201602-03670C

21. Jung $B$, Moury $P H$, Mahul $M$, de Jong A, Galia F, Prades A, et al. Diaphragmatic dysfunction in patients with ICU-acquired weakness and its impact on extubation failure. Intensive Care Med. 2016;42(5):853-861. [PubMed]

22. Ali ER, Mohamad AM. Diaphragm ultrasound as a new functional and morphological index of outcome, prognosis and discontinuation from mechanical ventilation in critically ill patients and evaluating the possible protective indices against 
VIDD. Egyptian J Chest Diseases Tuberculosis. 2017;66(2):339-351.

23. Li C, Li X, Han H, Cui H, Wang G, Wang

Z. Diaphragmatic ultrasonography for predicting ventilator weaning: A meta-analysis. Medicine (Baltimore). 2018;97(22):e10968. [PubMed] [Free full text] DOl:10.1097/ MD.0000000000010968

24. Vassilakopoulos T, Petrof BJ. Ventilator-induced diaphragmatic dysfunction. Am J Respir Crit Care Med. 2004;169(3):336-341. [PubMed]

25. Anzueto A, Peters JI, Tobin MJ, de los Santos R, Seidenfeld JJ, Moore $G$, et al. Effects of prolonged controlled mechanical ventilation on diaphragmatic function in healthy adult baboons. Crit Care Med. 1997;25(7):1187-1190. [PubMed]

26. Hermans G, Agten A, Testelmans D, Decramer M, Gayan-Ramirez G. Increased duration of mechanical ventilation is associated with decreased diaphragmatic force: a prospective observational study. Crit Care. 2010;14(4):R127. [PubMed] [Free full text]

27. Vivier E, Mekontso Dessap A, Dimassi S, Vargas F, Lyazidi A, Thille AW, et al. Diaphragm ultrasonography to estimate the work of breathing during non-invasive ventilation. Intensive Care Med. 2012;38(5):796-803. [PubMed] DOI: 10.1007/s00134-0122547-7

28. American Thoracic Society/European
Respiratory Society. ATS/ERS Statement on respiratory muscle testing. Am J Respir Crit Care Med. 2002;166(4):518-624. [PubMed]

29. Fadaii A, Amini SS, Bagheri B, Taherkhanchi B. Assessment of Rapid Shallow Breathing Index as a Predictor for $W$ eaning in Respiratory Care Unit. Tanaffos. 2012;11(3):28-31. [PubMed] [Free full text]

30. Pirompanich P, Romsaiyut S. Use of diaphragm thickening fraction combined with rapid shallow breathing index for predicting success of weaning from mechanical ventilator in medical patients. J Intensive Care. 2018;6:6. [PubMed] [Free full text] 